
\title{
R Research Sourere \\ Optimization of DMD-based independent amplitude and phase modulation: a spatial resolution and quantization
}

Alexandra Georgieva ( $\sim$ georgieva@itmo.ru )

ITMO University

Andrey Belashov

loffe Institute

Nikolay Petrov

ITMO University

\section{Research Article}

Keywords: digital micromirror device, wavefront modulation, digital holography, spatial resolution, image quantization

Posted Date: October 21st, 2021

DOl: https://doi.org/10.21203/rs.3.rs-966825/v1

License: (c) (i) This work is licensed under a Creative Commons Attribution 4.0 International License. Read Full License 


\title{
Optimization of DMD-based independent amplitude and phase modulation: a spatial resolution and quantization
}

\author{
Alexandra Georgieva ${ }^{1, *}$, Andrey V. Belashov ${ }^{2}$, and Nikolay V. Petrov ${ }^{1}$ \\ ${ }^{1}$ Digital and Display Holography Laboratory, Kronverksky 49, ITMO University, St. Petersburg, 197101, Russia \\ ${ }^{2}$ Ioffe Institute, 26 Politekhnicheskaya, St. Petersburg, 194021, Russia \\ *georgieva@itmo.ru
}

\begin{abstract}
The paper presents the results of a comprehensive statistical study on the optimization of the optical system for independent amplitude and phase wavefront manipulation, which is implemented on a binary digital micromirror device. Based on a statistical analysis of the data, an algorithm for selecting parameters (carrier frequency of binary pattern, and aperture for first-order diffraction filtering) that ensures the optimal quality of the modulated wavefront was developed. This algorithm takes into account the type of modulation, i.e. amplitude, phase, or amplitude-phase type, the size of the coded distribution, and its requirements for spatial resolution and quantization. The results of this study will greatly contribute to modulated wavefront quality improvement in various applications with different requirements to spatial resolution and quantization.
\end{abstract}

\section{Keywords}

digital micromirror device; wavefront modulation; digital holography; spatial resolution; image quantization

The synthesis of wavefronts with known characteristics has gained the interest of many researchers in the field of photonics. Some of the applications of wavefront shaping are high-resolution microscopy ${ }^{1}$, laser beam shaping ${ }^{2,3}$, scattering media characterization $^{4-6}$, holographic displays ${ }^{7}$, quantum cryptography ${ }^{8}$, metrology $^{9}$, compressed sensing ${ }^{10}, 3 \mathrm{D}$ bioprinting and lithography ${ }^{11}$. To date, there exists a range of static and dynamic wavefront modulators, such as diffraction optical elements ${ }^{12}$, metasurfaces ${ }^{13}$, adaptive optical elements ${ }^{14}$, which provide the possibility to operate with the amplitude, phase, or polarization of the beam profile in a wide range of wavelengths ${ }^{15,16}$. Adaptive spatial light modulators with programmable precise control of the wavefront have become a valuable tool for various applications, e.g., in imaging systems ${ }^{17}$. Two major types of such devices can be outlined: liquid crystal-based spatial light modulators and micro-electromechanical systems (MEMS). The former includes such subtypes as transmissive liquid crystal, reflective liquid crystal on silicon, and ferroelectric liquid crystal. MEMS-based spatial light modulators are presented by a digital micromirror device (DMD), active micromirror matrix, and grating light valve ${ }^{18}$.

Each of the devices is characterized by the type of modulation, among which they can be distinguished: amplitudeonly, phase-only, and simultaneous amplitude-phase modulation, and each of the modulators types has its benefits and disadvantages ${ }^{18-20}$. The choice of the required device is determined by the peculiarities of the problem to be solved in a particular case. Several important characteristics of wavefront modulators can be highlighted: the speed of operation, modulation dynamic range, number and size of pixels, modulation efficiency. In applications where high speed is required and spatial resolution can be sacrificed to achieve high light modulation rates ${ }^{21}$, the use of DMD is preferable due to its high refresh rate (up to $22 \mathrm{kHz}$ ). In addition, DMD constructively assumes only binary modulation. Over the past few years, such devices have been actively used in various studies ${ }^{11,21}$, and commercial devices (e.g., holotomographic microscope HT-1H, developed by Tomocube ${ }^{\mathrm{TM}}$ ). Compared to other modulators, DMD has high switching speed, light modulation efficiency and fill-factor (90\%), and relatively low $\operatorname{cost}^{22-24}$. It provides a high enhancement factor in the task of focusing through the scattering medium ${ }^{19}$ or improving contrast and beam-shaping fidelity in optical imaging ${ }^{20}$. This is particularly relevant in biomedical applications where rapid processes are involved, modulation of ultrashort radiation, or the possibility of real-time measurement should be provided ${ }^{11,21,25}$. DMD consists of a CMOS-placed micromirrors array, each of which can have only two stable states: "On" $\left(+12^{\circ}\right)$ and "Off" $\left(-12^{\circ}\right)^{21}$. Each micromirror represents a single pixel of the projected image. In addition, the use of binary (1-bit) holograms is convenient in terms of data capacity, for example, for implementation in holographic displays ${ }^{26}$. Another advantage of binary holograms over grayscale holograms is also that they can be easily printed ${ }^{27}$.

Various approaches have previously been proposed for generating binary DMD-patterns or converting grayscale holograms 
into binary holograms, for instance, global and local thresholding methods ${ }^{28}$, iterative techniques ${ }^{29,30}$, error diffusion method ${ }^{31}$, the superpixel-based method ${ }^{32}$, off-axis computer-generated Lee holography technique ${ }^{33}$. The latter is an efficient and fast method, especially eligible for ultrafast radiation modulation ${ }^{34}$. In this method, the $1^{\text {st }}$ diffraction order Fourier filtering is utilized, commonly with an aperture in experimental settings, which affects spatial resolution. The number of available amplitude levels (or image quantization) depends on the number of pixels in the fringe period in DMD-generated pattern ${ }^{35}$. These parameters significantly affect the image quality. Various methods of quantization and resolution improvement were proposed. The study by Reimers et.al. ${ }^{36}$ has elucidated the optimal resolution obtained for the detection of objects with different requirements for spatial resolution in terms of hyperspectral imaging. The results reported by Zhang et. al. ${ }^{37}$ suggest that in single-pixel imaging, the quantization error caused by binarization can be eliminated by error diffusion dithering and a high ratio of upsampling. In their recent study, Chipala and Kozacki ${ }^{7}$ demonstrated the correlation between the dispersion of the DMD and the resolution of holographic images, and proposed the method of image quality improvement. However, the area of achieving maximum quantization and resolution values by optimizing the experimental setup and binarization parameters has not been explored in depth. Moreover, the influence of the type of modulation (amplitude, phase, or amplitude-phase), differences in the number of points in the encoded distribution, and cutoff frequency DMD have not been analyzed in extensive studies. Meanwhile, in this study we demonstrate that in dependence on the target complex wave parameters, particular criteria imposed on the modulated wavefront, different binary patterns are required for optimal wavefront modulation. In certain types of applications either spatial resolution or quantization of the target complex wave may be the most important characteristic. For instance, some imaging applications of wavefront modulation, such as successful data transfer, targeted fluorescence proteins activation/desactivation, optogenetic stimulation, structured illumination fluorescence microscopy ${ }^{38-41}$, require the generation of amplitude or/and phase distributions with high spatial resolution. On the other hand, when the application of the wavefront modulation system is aberration correction ${ }^{42}$ or generation of uniform amplitude distribution, the quantization of both phase and amplitude distribution should be taken into account to provide high-performance wavefront modulation with high dynamic range and discretization ${ }^{43,44}$. Moreover, in certain applications, such as the implementation of ptychographic approaches ${ }^{45}$ both of these parameters may be important and a certain trade-off between these characteristics should be found in each particular case.

Herein, in the given work, we developed and implemented a numerical model for simulation of DMD-based complex wave modulation and evaluation of its accuracy. A statistical study was performed revealing the dependence between the wavefront modulation quality, and such parameters of the target wave as its size, modulation type (phase / amplitude / amplitude-phase), and the requirements of spatial resolution and quantization. The algorithm was developed for determination of the optimal modulation parameters parameters (the carrier frequency of the binary pattern and the aperture for the first diffraction order filtering). In addition, experimental validation of the proposed optimization method was performed. It was found that for applications requiring high spatial resolution, it is necessary to sacrifice the number of amplitude gradations to increase the filtration aperture in Fourier domain. The work also clearly demonstrates that for applications where it is important to preserve quantization levels, it is possible to increase the binary fringe period of DMD-pattern.

The paper is organized as follows: In Sections 2.1 and 2.2, an experimental setup and basic principles of independent amplitude and phase wavefront modulation using DMD are described. Subsection 2.3 describes the criteria for modulated wavefront quality estimation. In Section 3.1 we consider the problem of wavefront modulation accuracy maximization by the variation of spatial filter aperture size and carrier frequency of binary digital hologram. The impact of these input parameters on the spatial resolution and amplitude/phase distribution quantization is considered and discussed. Subsection 3.2 demonstrates the results of the statistical study on the modulation type and target distribution size for the optical system optimization for independent amplitude and phase wavefront manipulation. Section 4 shows experimental results of wavefront manipulation using random and optimized DMD pattern and in conclusion the major results of the study are briefly summarized.

\section{Methods}

\section{Experimental setup}

The experimental setup is presented by the Mach-Zender interferometer with DMD and 4-f lens system in the object beam (Fig. 1).

This setup provides an opportunity to modulate the wavefront using DMD, as well as to evaluate its modulation quality by image reconstruction with off-axis digital holograms. DMD was operated using controller DLPC900 (modulation frequency $9 \mathrm{kHz}$ ). The laser beam with the wavelength of $532 \mathrm{~nm}$ was increased in diameter by the beam expander BE, then it was divided into object and reference waves by the beam splitter $\mathrm{BS}_{1}$. The object beam was incident on the DMD (Texas Instrument Light Crafter DLP6500FYE in our case with $1920 \times 1080$ micromirrors with a size of $7.56 \mu \mathrm{m}$ ), where the corresponding pattern was displayed on the matrix. Then it was focused with the lens $L_{1}$ to the Fourier plane, where the $1^{s t}$ diffraction order was separated by the spatial filter SF with aperture size scaleable along the $x$ coordinate. Afterward, the lens $\mathrm{L}_{2}$, which together with $L_{1}$ forms telecentric $4 f$-system, collimated the radiation. The desired field distribution was formed in the target plane TP 


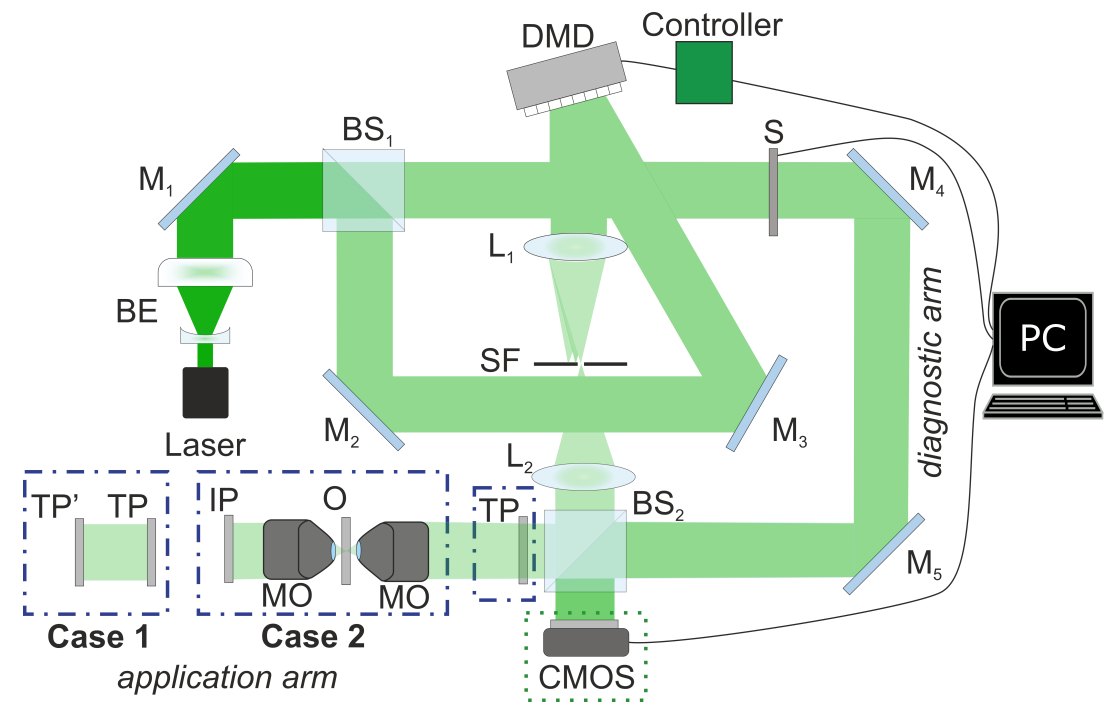

Figure 1. Experimental setup. $B E$ are beam expanders, $M_{1-5}$ are the mirrors, $S$ is a shutter, $B_{1-2}$ are the beam splitters, $\mathrm{L}_{1-2}$ are lens, $\mathrm{SF}$ is the spatial filter, TP is the target plane, CMOS is the matrix detector, MO are objectives, IP is an image plane, $\mathrm{O}$ is an object.

after the second beamsplitter $\mathrm{BS}_{2}$. The presence of $\mathrm{BS}_{2}$ ensured the location of this plane simultaneously both at the end of the diagnostic arm, namely, in the sensor plane (CMOS) and in the application arm.

The beamsplitter $\mathrm{BS}_{2}$ enabled performing both the simultaneous monitoring of the wave, independently modulated in amplitude and phase by off-axis digital holography using CMOS sensor in the monitoring arm (indicated with green dotted frame in Fig. 1) and utilized the modulated wave for any research purposes in the application arm (indicated with blue dash-dotted frame in Fig. 1. Plane reference wave (when the shutter $S$ is opened) passes through the mirrors $M_{4}$ and $M_{5}$ and enabled detection of formed amplitude and phase distributions in the application arm. Holographic monitoring was implemented in the following way: wave, modulated with DMD, and a plane reference wave incidence on the sensor of CMOS camera at a small angle, thus generating an off-axis digital hologram. Then we used the local least square estimation algorithm ${ }^{46-48}$ for complex wave reconstruction.

It should be noted that some applications may require to obtain the target wavefront of a specific structure beyond the target plane (TP). Such a complex wave located in arbitrary plane may be engineered precisely by means of an analysis of the resulting field at TP in the diagnostic arm and solving the diffractive equations, which describe the wavefront propagation beyond the TP in backward direction ${ }^{49}$ (case 1 in Fig. 1). Then, this information should be taken into account in the feedback loop: the calculated wavefront distribution is set as the target one, taking into account possible error correction procedures to minimize the mismatch between the target and actually generated wavefront in TP. Then, after the wavefront synthesis in TP, its further physical diffraction propagation forms the initially desired wavefront at a certain plane.

The alternative is not to propagate wavefront numerically, but to image TP further by an additional telecentric system with custom magnification ${ }^{50}$ (case 2 in Fig. 1). It is also necessary to mention that independent values in amplitude and phase distributions may be obtained in the TP (or in the corresponding image plane, as a result of the projection of the 4- $f$ telecentric system) only, since due to diffraction the phase distribution of a coherent wave affect the amplitude distribution and vise versa.

\section{Principles of binary complex wavefront modulation}

In this subsection, the basic principles of independent amplitude and phase modulation of the wavefront using DMD generated binary off-axis digital hologram are considered and discussed. One of the possible approaches for independent amplitude and phase modulation was proposed by Lee et al. ${ }^{33}$ is to generate a binary pattern and "reconstruction" of the synthetic off-axis digital hologram.

It is known that fringes in an analog hologram can be used to change the phase and amplitude of the incident light wave, thus producing an image of the recorded object. Similar to analog holography, the variation of binary fringe parameters such as width and periodicity in the formed binary DMD pattern, enables manipulation of amplitude and phase distributions. The approach to obtaining independent amplitude-phase modulation from binary amplitude modulation is based on the spatial filtering of the wave in one of the diffraction orders arising from the reflection of an incident wave from DMD. It can be 
conducted, for example, by spatial filtration of this diffraction order if the binary pattern is an off-axis hologram with high enough carrier frequency.

Experimentally, it is usually implemented by Fourier transform performed using a concave lens (L1), as demonstrated in Fig. 1. Filtration of the first diffraction order using adjustable iris aperture (SF), while inverse Fourier transform by another concave lens L2 enables the reconstruction of the target wave modulated by DMD.

The spatial frequency of the binary hologram is calculated using the carrier frequency and phase change as follows ${ }^{33}$ :

$$
\begin{aligned}
& v_{x}(x, y)=k_{x}+\frac{1}{2 \pi} \frac{\partial \phi(x, y)}{\partial x} \\
& v_{y}(x, y)=k_{y}+\frac{1}{2 \pi} \frac{\partial \phi(x, y)}{\partial y}
\end{aligned}
$$

where $k_{x}$ and $k_{x}$ are carrier frequencies in the $x$ and $y$ directions, which are inversely proportional to the specified fringe period due to the wavefront tilt. The second summand refers to the spatial frequency of the wave with target phase distribution.

DMD-pattern $h(x, y)$ was formed using the limiter to obtain amplitude hologram binarization ${ }^{33}$ :

$$
h(x, y)=\left\{\begin{array}{lll}
1 ; & \left|\left(\left[\frac{\phi(x, y)}{2 \pi}+\left(k_{x} \cdot x+k_{y} \cdot y\right)\right] \quad \bmod 1\right)-0.5\right| \leq \frac{\operatorname{asin}|A(x, y)|}{2 \pi} \\
0 ; & \text { otherwise }
\end{array}\right.
$$

where $A(x, y)$ is the target amplitude, $\phi(x, y)$ is the target phase, $k_{x}$ and $k_{y}$ are the carrier frequencies in DMD pixels of the binary off-axis hologram in the $x$ and $y$ direction. The left part of the inequality (2) is responsible for the encoding of the phase distribution, while the amplitude distribution is encoded by the right part. To show this segregation of amplitude and phase parts, we consider DMD-patterns simulated for amplitude-type, phase-type, and amplitude-phase modulations (Fig. 2). By the type of modulation in this case we mean an amplitude-only, phase-only, or simultaneous independent amplitude and phase wavefront variation in the $T P$ plane.

The first example of varying amplitude (2D Gaussian distribution) and constant phase distributions is presented in Fig. 2 (ac). The target amplitude image (Fig. 2 (a) and the simulated binary pattern (Fig. 2 (b)) demonstrate how the complex wave amplitude can be modulated by the variation of the relative amount of DMD pixels at On-state (the On-pixels). The plot of the phase part of inequality, indicated by the red line, clearly shows how the inequality (2) provides an opportunity to readily synthesize a binary pattern for a given amplitude distribution. The right (amplitude) and left (phase) parts of the inequality are presented by red and blue lines, respectively. While in this case, the phase distribution is constant, the left part of the inequality representing the phase distribution, is a simple periodical function due to the linear increase of $\frac{\phi(x, y)}{2 \pi}+\left(k_{x} \cdot x+k_{y} \cdot y\right)$ term and " $\bmod 1$ " operation. However, the target amplitude image is changed along $x$ coordinate (red line), thus varying the relative amount of the On and Off pixels. If the amplitude part is larger than the phase part, the pixel is On, otherwise it remains Off. In this case, the ratio between the On pixels and the total number of pixels per one period (the On pixel occupancy coefficient $\eta$ ) is changed from 0.3 to 0.5 while the target amplitude increases approximately from 0.13 to 0.25 .

Due to the constant phase distribution, the carrier frequency, as well as the value of spatial frequency of the binary hologram here is constant and equals $\frac{2 \pi}{12}$. Subsequently, the fringe period is 12 DMD pixels (indicated with black arrows in Fig. 2 (c)). The phase-only variation is demonstrated with the target spherical phase distribution and constant amplitude. Fig. 2 (d-f) show the target phase distribution (Fig. 2 (d)), the simulated DMD pattern (Fig. 2 (e)) and the plotted functions of DMD pattern pixels, amplitude part and phase part (Fig. 2 (f)) of inequality (2) in the case of phase-only modulation. In this example, the right part of the inequality (2) is constant due to the absence of amplitude modulation. However, variation of the target phase along $x$ axis results in the change of $\frac{\phi(x, y)}{2 \pi}+\left(k_{x} \cdot x+k_{y} \cdot y\right)$ slope and alteration of the phase part periodicity and spatial frequency of binary hologram. The black arrows in Fig. 2 (f) demonstrate how the phase variation results in the change of the fringe period from 29 to 20 pixels. At the same time, $\eta$ is constant among the binary hologram and equal to 0.5 .

Therefore, phase modulation is achieved by the variation of the binary fringe period, while the amplitude modulation is obtained due to the variation of the On pixel occupancy coefficient. Since it is possible to separately vary these two parameters in each local area of the image, the independent manipulation of the phase and amplitude distributions can be achieved.

An example of independent modulation of both amplitude and phase parts of the complex wavefront is presented in Fig. 2 (g-i). It can be noted that both fringe periodicity and the On pixel occupancy coefficient are changed due to amplitude and phase variation.

\section{Modulation error assessment}

Quantitative assessment of the modulated wavefront quality can be achieved by calculation of root mean square error (RMSE) between true target distribution and the resulted modulated wavefront. To perform optimization and find the optimal parameters, 


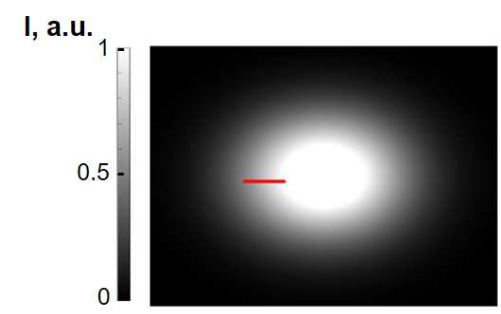

(a)

$\varphi, \mathrm{rad}$

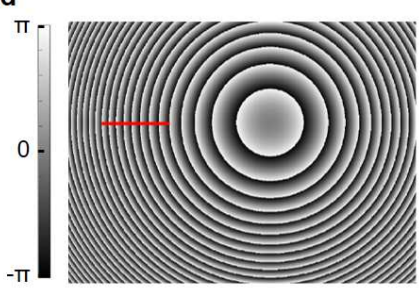

(d)

$\varphi$, radll, a.u.

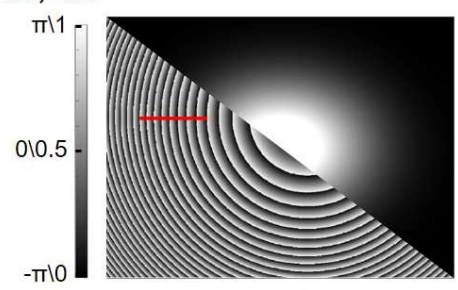

(g)

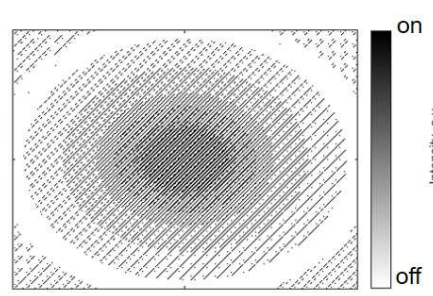

(b)

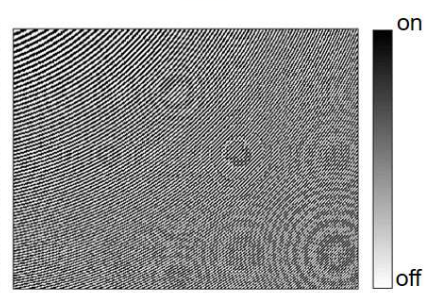

(e)

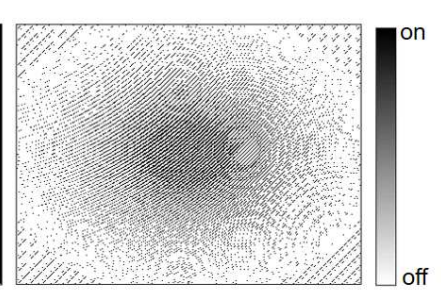

(h)

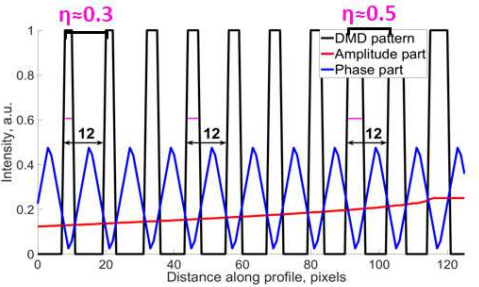

(c)

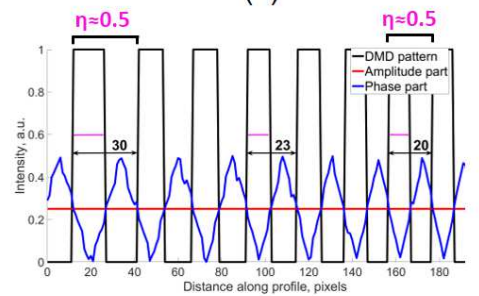

(f)

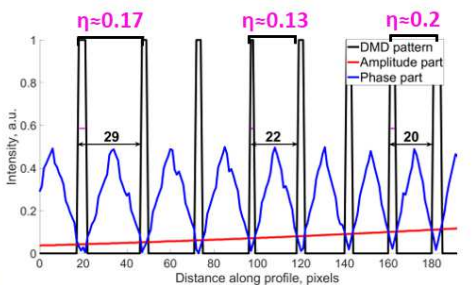

(i)

Figure 2. Demonstration of independent amplitude (a-c), phase (d-f), and amplitude-phase (g-i) modulation. Target amplitude (a), phase (d), amplitude-phase (g) distribution. The red line indicates the coordinates of plotting in (c,f,i); (b,e,h) Resulting DMD pattern (carrier frequency $\left.\frac{2 \pi}{12}\right) ;(\mathrm{c}, \mathrm{f}, \mathrm{i})$ the plot of DMD pattern function (black line), phase part (blue line) and amplitude part (red line).

we numerically simulated DMD-based target wavefront modulation with various carrier frequencies and filtration aperture sizes and calculated RMSE of the target amplitude or phase distributions using the following equation:

$$
R M S E=\sqrt{\frac{1}{n} \sum_{i=1}^{n}\left(x_{t}-x_{r}\right)^{2}},
$$

where $n$ is the number of pixels of distribution, $x_{t}$ is the value of target amplitude or phase at some pixel, $x_{r}$ is the value of resulting amplitude or phase at some pixel. Minimization of the calculated error enables obtaining the optimal parameters of DMD-generated binary pattern for a particular target complex wave.

To investigate the dependence of the modulation quality on the major experimental scheme parameters, each of these images was encoded with the aperture size ranging from 1 to 400 pixels and the fringe period varying from 1 to 100 pixels. Note that in our numerical simulation size of the spatial filtration aperture is in relative values (pixels within the Fourier domain). Physical value of the spatial aperture in experimental work should be recalculated taking into account focusing distance of $L_{1}$ (see Fig. 1) and DMD physical size. The location of every i-th pixel of DMD in the focal plane of $L_{1}$ can be calculated as $i \frac{f_{1} \lambda}{N \Delta_{p}}$, where $f_{1}$ is focal distance of $L_{1}, \lambda$ is wavelength, $N$ is linear matrix size and $\Delta_{p}$ is pixel size. For each of the obtained images, its RMSE from the target wave was calculated and represented as a 2D pseudocolored surface (see Fig. 3). Analysis of such a 2D wavefront modulation error map makes it possible to determine the optimal parameters of the complex wave modulation.

The resulting amplitude and phase wavefront modulation error maps are separated on high and low error areas with a hyperbola-type curve, above which all resulting distributions have critical defects in the form of interference fringes, caused by the overlay of two (or more) adjacent diffraction orders into the aperture. Utilizing small apertures also leads to defects of the modulated wavefront due to excessive spatial filtering resulting in the decrease of spatial resolution and image blurring. The area of high apertures and low fringe periods also leads to defects expressed in the form of sharp shade transitions due to the 

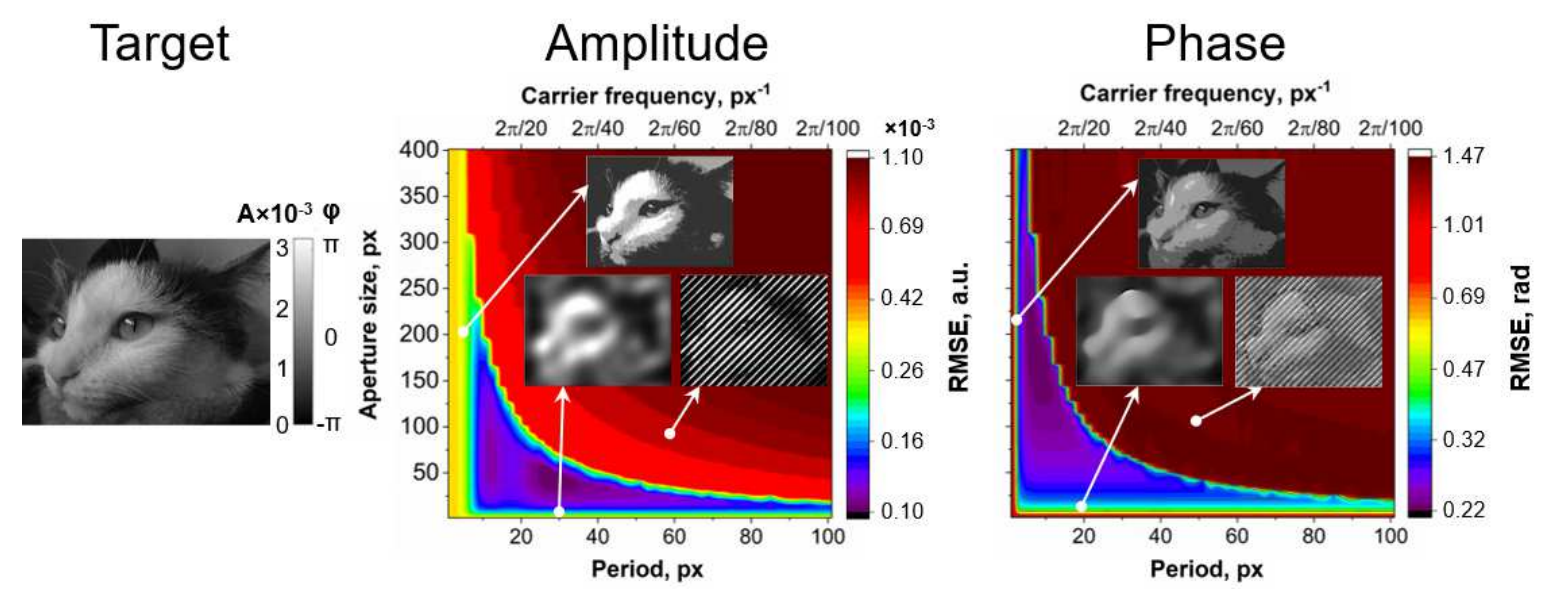

Figure 3. Amplitude and phase modulation RMSE maps dependent from aperture size and fringe period for the "cat" object. Insets: obtained intensity distributions at the points indicated by the markers. Target distribution is shown on the left.

limited number of gradations. The detailed discussion of the optical system parameters influence on the wavefront quality is presented in Suppl. 1. Therefore, to determine the optimal modulation parameters, it is necessary to consider several factors, which will be discussed below.

In case of simultaneous amplitude and phase modulation the modulation error can be calculated as follows: $\delta=1-F$, where $F=\left|E_{\text {target }}^{*} E_{\text {obtained }}\right|^{2}, E_{\text {target }}$ is a target field, $E_{\text {obtained }}$ is the field obtained after DMD modulation ${ }^{32}$.

\section{Results of numerical study on aperture size and carrier frequency optimization}

\section{Target diversity study}

This section discusses the main factors influencing the choice of optimal parameters, i.e., aperture size and fringes period or carrier frequency of binary hologram, to ensure the best quality of the modulated complex wave distributions.

Let us consider the encoding of few representative target distributions containing a different range of spatial frequencies and intensity levels: "circles", phase object characterized by high and low spatial frequencies content (mixed-frequency phase object $^{51}$ and 2D Gaussian distribution. The object "circles" is used as a demonstration of the small elements' content influence on optimal aperture size and fringe period selection. The object 2D Gaussian distribution is used to demonstrate the example of high image quantization and low requirements to spatial resolution. A Mixed-frequency object is an example of complex distribution with almost equal requirements to spatial resolution and quantization. Figure 4 shows the modulation error maps of amplitude and phase in the case of amplitude-only and phase-only field variations, respectively, for these objects. The insets show the examples of the obtained intensity and phase distributions at the optimal parameters, corresponding to the minimum error. Note that all the phase patterns suggest modulation within the range from $-2 \pi: 2 \pi$ values. Strong variation of the phase value range may result in a significant change of the parameters for optimal complex wave modulation.

At first, we will describe the extreme examples, requiring either high spatial resolution or high quantization. For complex wave modulation with the "circles" patterns, it is important to achieve the maximum possible aperture size to provide sufficient spatial resolution. The target image includes repetitive circles of several fixed sizes. Simultaneously, for the 2D Gaussian example, a decrease in the distance between diffraction orders and the aperture size does not significantly deteriorate the image quality due to the absence of small details in this image. As shown in Fig. 4 (a,b), the minimal error area is local, and the global minimum corresponds to large aperture size and small fringe period. In the case of 2D Gaussian distribution, the high error of amplitude and phase modulation corresponds only to small fringe periods (high carrier frequencies) (Fig. 4 (c,d)). The minimum error of wavefront modulation can be achieved in the case of small aperture size and large fringe period.

In the case of mixed-frequency object parameters, corresponding to optimal complex wave modulation significantly depends on the type of modulation - amplitude or phase (Fig. 4 (e,f)). In both cases, the minimum modulation error corresponds approximately to the average size of the aperture and period but for amplitude-type modulation, the minimum is shifted towards higher periods, and for phase modulation - towards higher aperture sizes. This can be explained by the presence of both high spatial frequencies, i.e., requirements for spatial resolution, and the presence of low spatial frequencies, the main part of the distribution is occupied by the band corresponding to the low-frequency elements with corresponding phase values $\pi$ and $-\pi$. 


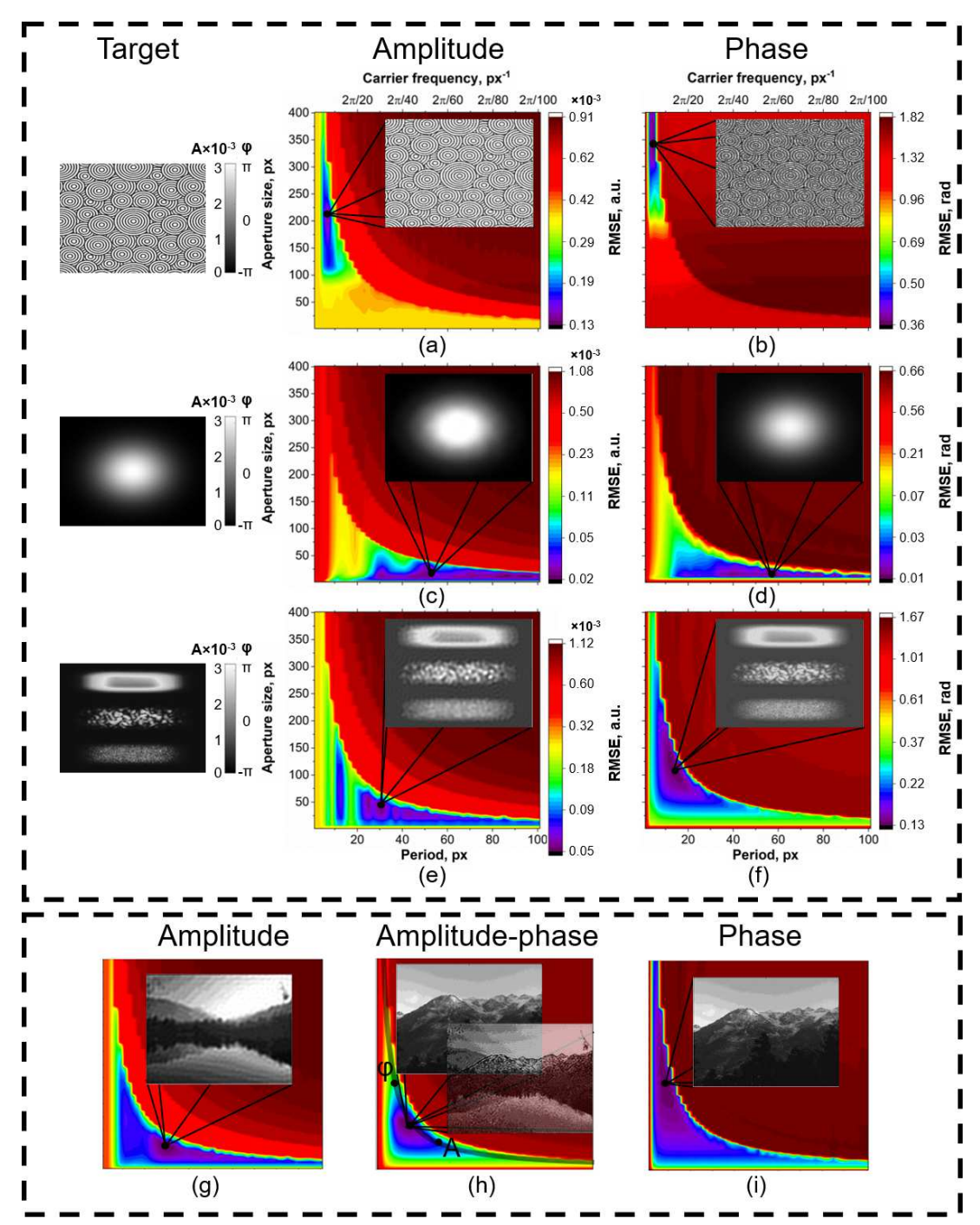

Figure 4. Wavefront modulation error maps for amplitude, phase and amplitude-phase modulation dependent on aperture size and fringe period for different objects. Insets: enlarged fragments, obtained intensity and phase distributions at the minimum RMSE points indicated by the markers. The upper frame shows amplitude (a,c,e) and phase (b,d,f) modulation error maps for objects "circles", 2D Gaussian distribution and mixed-frequency phase object. Target distributions are shown on the left. The lower frame shows amplitude (g), phase (h) and amplitude-phase (i) modulation error maps. The minimal RMSE for amplitude-phase modulation lies approximately in the medium of amplitude and phase optimal parameters.

Until now we considered the cases when only phase or only amplitude modulation took place. However, our DMD-based modulation system allows for simultaneous and independent amplitude-phase modulation, which provides unique opportunities for light manipulation in comparison with other techniques, e.g., based on liquid crystal-based spatial light modulators. Therefore the case of simultaneous amplitude and phase modulation should be considered as well. Objects in both amplitude and phase were selected to investigate independent amplitude-modulation modulation. The amplitude error, phase error, and modulation error together with the resulting intensity and phase distributions are shown in Fig. 4 (g-i).

Figure 4 (g) shows that for the amplitude modulation of a complex object the area of minimum errors is shifted towards larger periods. The phase modulation optimum of the other complex distribution is located in the area of larger apertures (Fig. $4(\mathrm{~h})$ ). The modulation error minimum for independent amplitude-phase modulation is located in the middle of the graph (Fig. 4 (i), i.e., approximately in the middle between the optimums for amplitude and phase modulation separately. Consequently, in the case of encoding of complex waves requiring both spatial resolution and quantization, it is necessary to choose the average values of the aperture size and fringe period. 
In general, the area of minimal error for all distributions is below the hyperbola-type curve, defined by the expression aperture $=\frac{a}{\text { period }}$, where $a$ depends on the image size and the parameters of the optical system. Hence, regardless of the type of distribution, the optimal ratio must be sought below this curve. Furthermore, optimal parameters for amplitude-phase modulation lie approximately in the middle of the optimal parameters of amplitude-only and phase-only modulation for the distributions. Therefore, we will examine in more detail the dependence of the location of the optimum on the modulation type.

\section{Statistical study on modulation type and target size}

The presented above results demonstrate a strong correlation between image quality and aperture size and carrier frequency or fringe period. In dependence on the target wavefront parameters, various binary patterns with different carrier frequencies should be generated. In this section, the influence of the modulation type will be considered in the context of a statistical study of different types of images.

As it was shown in Fig. 4, the minimum value of RMSE is located in some area of the error map, dependent on distribution type. To determine the area of minimum modulation error it is also necessary to consider the dependence of the position of optimums on the type of modulation. We performed a numerical simulation of amplitude and phase modulation and found the minimum values of wavefront modulation error maps. A dataset consisting of 591 images $^{52}$ was implemented. The distribution of the minimum error points is shown in the Fig. 5 (a).

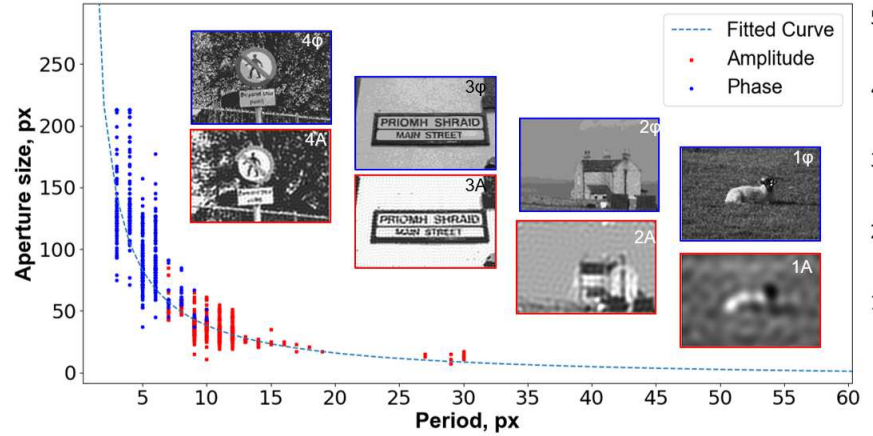

(a)

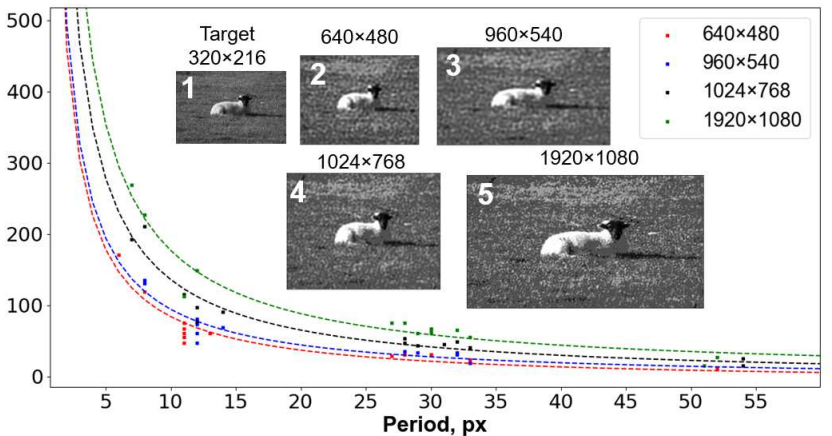

(b)

Figure 5. Minimal RMSE graph for amplitude-type and phase-type modulation. Approximation curves are plotted using specified points and have the hyperbola-type form. (a) Minimal RMSE for dataset ${ }^{52}$ are indicated with red (amplitude-type) and blue (phase-type) dots. The insets show amplitude (red rectangles) and phase (blue rectangles) distributions, obtained using parameters which presented above each image. (b) Minimal RMSE points for 10 different objects and different sizes, equal to DMD matrix sizes for amplitude- and phase-type modulation. The insets show amplitude distributions, modulated using different DMD display resolution which presented above each image.

As can be seen from Fig. 5 (a), for amplitude-type modulation (red dots), the minimum error values lie in the area of large periods and small apertures, while the minimum error values for phase-type modulation (blue dots) are located in the area of the graph with large aperture size. This effect can be explained by the influence of the distribution of diffraction orders in the Fourier plane. In the case of amplitude-type modulation, only the intensity of the diffraction order varies, but not its form and direction. Phase-type modulation involves changing the form and direction of the first diffraction order, which is especially critical for significant drops in the given phase distribution (e.g., from 0 to $2 \pi$ ). Thus, for encoding only the amplitude distribution, it is possible to increase the period of the binary pattern to achieve the required level of quantization, with small requirements to the size of the aperture. At the same time, coding the phase distribution, especially containing high spatial frequencies and phase differences, requires increasing the aperture size, which entails the need to reduce the period to ensure, among other things, a sufficient level of quantization. As a consequence, the order of phase modulation errors is much higher than the order of amplitude modulation errors.

The few points corresponding to amplitude modulation with the binary fringe period of more than 25 pixels are optimums for images with frequent fluctuations in amplitude or phase (e.g., the grass in the inset image $1 A$ in Fig. 5 (a)). It is also seen that such a distribution is not resolved by amplitude modulation. This can be the result of the fact that such tiny details of the images a-priori can not be resolved within the given optical system. The insets also demonstrate the amplitude and phase distributions obtained at the indicated points. It can be seen that depending on the type of image (spatial resolution and quantization requirements), the optima are shifted along the hyperbola-type curve which represents the inverse dependence 
between quantization and spatial resolution. However, for distributions with small and frequent fluctuations, it is not possible to perform amplitude modulation due to the insufficiency of encoding pixels in a period. Therefore, we decided to study the dependence between the optimum point location and the size of the modulator matrix in detail.

In the study of finding the optimal parameters for images with different spatial resolution and quantization requirements, it was shown that the area of small modulation errors is below the hyperbola-type curve. The amplitude distributions of small size and frequent small fluctuation were unresolvable. We consider how the DMD matrix size affects the modulation quality in the context of spatial resolution and quantization.

In addition to analysis of the experimental parameters optimal for complex wave modulation, we would like to discuss the impact of the target image size impact onto the global minima position on the wavefront modulation error maps. To do so, we performed a set of additional numerical experiments with different sizes of the same target amplitude and phase distributions, corresponding to various DMD array sizes available on the market. Four DMDs with different display resolution were considered - DLP230GP $(960 \times 540)$, DLP7000 $(1024 \times 768)$, DLP6500 $(1920 \times 1080)$, and the first DMD ${ }^{53}(640 \times 480)$, all of which were developed by Texas Instruments ${ }^{\mathrm{TM}}$. Wavefront modulation error maps were constructed for 10 images and optimal modulation parameters were found and plotted in Fig. 5 (b).

The dotted line shows the approximation curves, which demonstrate the dependence of the position of the optimum relative to the same images modulated by different DMDs. Figure 5 (b) shows that the approximation curve has a larger coefficient with increasing matrix size, i.e., with increasing matrix size the aperture size and fringe period on the binary pattern increases. Depending on the type of image, the position of the optimum varies along the curve. In addition, the DMD cutoff frequency also affects the image quality, especially for amplitude modulation. Since amplitude modulation is determined by pixel occupancy rate, when using a small matrix (e.g., $640 \times 480$ ) for a distribution that has frequent amplitude variation (grass in Inset 1 in Fig. 5 (b), Inset 1A in Fig. 5 (a)) the fringe period must be large enough to ensure that all amplitude fluctuations are encoded. In other words, to encode frequent changes in amplitude in a binary pattern, frequent changes in the pixels that are on and off are observed, which is impossible with insufficient display resolution. In addition, in this case, a much smaller aperture size is required, which leads to a decrease in spatial resolution. Nevertheless, using a DMD with larger matrices allows encoding smaller amplitude fluctuations for the same distributions (Insets 3-5 in Fig. 5 (b)). At the same time, the phase modulation is encoded by changing the slope of the binary pattern fringes. Therefore, it is not necessary to utilize a large fringe period to encode small phase fluctuations. Thus, the DMD matrix size is directly related to the quality of the obtained amplitude images, however, the phase modulation, in this case, is less dependent on the cutoff frequency (Inset 1A and $1 \varphi$ in Fig. 5 (a)).

Since the distance between the diffraction orders in the frequency space is proportional to the carrier frequency of the binary pattern as well as to the pixel size, the pixel size in numerical simulation is assumed to be 1 , at carrier frequencies $k_{x}=k_{y}=k$, respectively, to calculate the hyperbola coefficient $a$ it is necessary to count the product of the DMD matrix diagonal to the pixel size. Thus, this curve will be an envelope, above which all parameters can be disregarded since the interference of neighboring diffraction orders will be observed in the detection plane.

\section{Experimental results}

In the presented above sections, the factors affecting the quality of amplitude or phase images are discussed. On this basis, for the experimental validation of numerical simulation, optimization of the parameters of the binary pattern and the experimental setup was conducted.

Experimental confirmation of the image reconstruction quality dependence on aperture size was performed on the example of phase-only modulation of the USAF 1951 test chart. The experimental setup is described in Subsection . This object is usually applied for the determination of optical systems spatial resolution and can be successfully used for the demonstration of aperture size impact on the spatial resolution of the modulated wave. Since this object should be reconstructed with the highest possible spatial resolution, the setup parameters and the binary pattern were selected according to the optimization results (Section, ). First of all, the distance between adjusting diffraction orders can be calculated as the ratio of the diagonal of the DMD matrix to the carrier frequency of the pattern. Then, as we perform phase-type modulation, the optimum will be located in the area of larger apertures and smaller periods. Since many phase gradations are not required in this case, it is sufficient to proceed from the capabilities of the particular experimental setup. In our case, the minimum period of the binary pattern was 7 pixels. Therefore, the distance between diffraction orders is approximately $2.4 \mathrm{~mm}$. We performed three experiments with different sizes of aperture, presented in Fig. 6.

To confirm the accuracy of the selected parameters, the aperture size for filtration was changed in the $x$ axis. The micrometeradjusted monochromator slit was set in the Fourier plane to filter the first diffraction order. It allowed us to precisely vary the size of the filtration area along the $x$ coordinate. The variation of only a single coordinate spatial aperture enables the demonstration of the impact of the aperture size.

The results of phase distributions reconstruction at different filtering aperture sizes are shown in Fig. 6. Whereas the size was changed along the $x$ axis, image blurring using the insufficient aperture size is observed only in the horizontal direction 


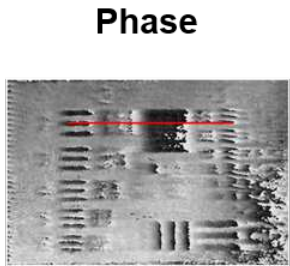

(b)

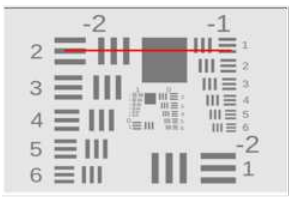

(a)

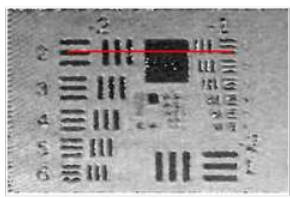

(d)

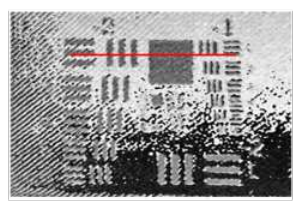

(f)
Cross-sections

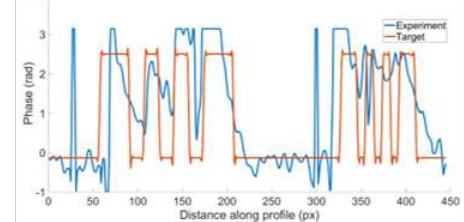

(c)

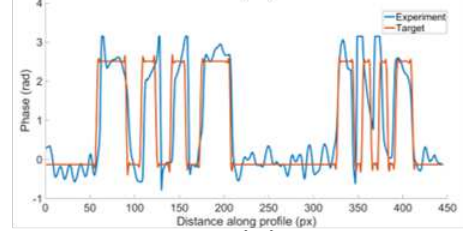

(e)

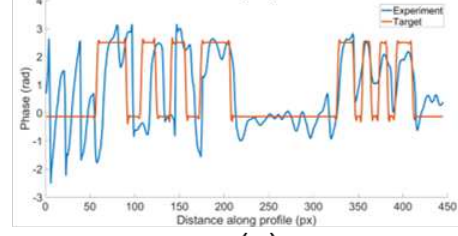

(g)

Figure 6. The results of phase distributions reconstruction with different filtering aperture sizes. (a) Target phase distribution; $(\mathrm{b}, \mathrm{d}, \mathrm{f})$ reconstructed phase distributions with aperture size $1.5 \mathrm{~mm}, 2.25 \mathrm{~mm}$ and $3.0 \mathrm{~mm}$, respectively. The red lines indicate the coordinates of cross-sections; $(\mathrm{c}, \mathrm{e}, \mathrm{g})$ the cross-sections of reconstructed phase distributions and target distribution.

(Fig. 6 (a)). Figure 6 (e) shows the image deterioration due to adjacent diffraction orders passing through the filtration aperture. As can be seen in Fig. 6 (c), the optimal aperture size allowed us to reconstruct the phase distribution with minimal error and high

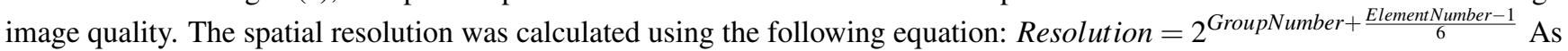
it can be seen from Fig. 6 (d), Group Number was "-1", and Element Number was "3". Real size USAF 1951 test chart to DMD pattern size conversion ratio was 13.8. Therefore, the estimated spatial resolution was $8.72 \mathrm{lp} / \mathrm{mm}$.

The cross-sections comparing the target and reconstructed distributions are provided (Fig. 6 (c,e,g)). In the case of the optimal aperture, an almost complete phase coincidence is observed (Fig. 6 (d)). For a small aperture, there are no sharp edges between the objects, which is demonstrated in Fig. 6 (b). For large apertures, the image corrupting fringes resulting from the adjacent diffraction orders passage can also be seen in the cross-section in Fig. 6 (f)).

The experimental results have demonstrated that the parameters of the binary pattern and aperture size, selected by the described above method allowed to reconstruct the phase with high image quality. The correlation between the aperture size and image quality was also confirmed.

\section{Discussion and conclusion}

Over the past few decades, wavefront manipulation by DMD has been widely used in light processing technology, despite the fact that DMD itself can be used for binary modulation of amplitude distribution only. The modulation of both amplitude and phase distribution is performed in two steps. Firstly, it is necessary to generate a binary pattern and project it on DMD. The pattern must be encoded in such a way that the wave reflected from DMD is divided into several diffraction orders, and the target distribution is contained in one of them. Secondly, this diffraction order is filtered by aperture, while others are blocked. Although there are various techniques for binarization of necessary patterns ${ }^{28-32}$, computer-generated Lee holography ${ }^{33}$ is one of the most reliable methods to perform fast and efficient generation of binary patterns.

As it was mentioned above, this method assumes the generation of binary patterns and filtration of the first diffraction order in the Fourier domain. The quality of the wavefront modulation is significantly affected by experimental parameters such as filtration aperture size and binary hologram carrier frequency. The variation of these two parameters enables the optimization of the quality of complex wave modulation and the achievement of either high spatial resolution or high image quantization. If the target complex wave requires high spatial resolution, carrier frequency, and filtration aperture size should be increased. However, target complex wave with gradually changing phase or amplitude values modulation requires a high quantization of 
target amplitude or phase distribution. Therefore, the considered parameters should be decreased to provide the best quality of wave modulation.

The obtained results show that different types of target amplitude and phase distributions require different experimental parameters (i.e. binary fringe period and filtration aperture size) in dependence on the image type. On the other hand, all the analyzed target amplitude and phase distributions demonstrate the best modulation quality when the mentioned experimental parameters values lie below the hyperbola-type curve. In addition, in general, amplitude-only modulation of the incident wavefront typically requires higher fringes periods, while phase modulation requires larger filtration apertures. Moreover, larger target images for wavefront modulation allow us to obtain better spatial resolution in terms of amplitude modulation.

To determine the optimal parameters for a particular case, it is necessary to consider several aspects of a target distribution. Initially, the size of the DMD matrix is taken into account. In the case where the binary pattern is generated with equal carrier frequencies in both directions, the limiting distance between diffraction orders is calculated. Thus, we can determine the limiting coefficient of the hyperbola, above which all parameters will be suboptimal due to the superposition of diffraction orders. Further, it is necessary to proceed from the type of modulation. For amplitude modulation to find the optimal parameters we need to consider only the upper part of the hyperbola, and in the case of phase modulation - only the lower part. Finally, it is necessary to determine the ratio of the need to preserve spatial resolution and quantization. In the case of the predominance of the need to detail small elements of the distribution, the area of optimum will be shifted towards large apertures and small periods. In contrast, if the need to preserve quantization (e.g., when compensating for aberrations) prevails, the optimum is shifted toward larger periods. One critical point is the technical aspect. For the realization of patterns with large periods, it is physically rather difficult to perform spatial filtering. Thus, the algorithm is as follows: (1) determine the critical values of apertures and periods according to the formula, above this hyperbola values are not considered; (2) type of modulation, for amplitude - above the hyperbola, for phase - below; (3) determine the ratio of spatial resolution and image quantization requirements, depending on the task; (4) for amplitude-phase modulation we take average values between certain parameters separately for amplitude and separately for phase modulation.

The correlations between quantization and carrier frequency, resolution and aperture size are particularly interesting considering the wide variety of applications utilizing DMD. For instance, DMD patterns with lower spatial frequencies are used to correct aberrations by increasing the fringe period and decreasing the filtering aperture. Laser processing ${ }^{14}$, optoacoustic microscopy ${ }^{19}$ or optical coherence tomography ${ }^{54}$ require increased accuracy and high spatial resolution, which can be achieved by increasing the filtering aperture and decreasing the fringe period of binary hologram. In the case when the target wavefront poses a slow variation of amplitude and phase distributions, e.g., cells or intracellular organelles, quantization of modulated complex wave becomes important. Thus, in applications of such type, it is impossible to sacrifice one parameter to improve another and it is necessary to maximize equally the spatial resolution and quantization of images.

In summary, the results reported here demonstrate that the achievement of the accurate wavefront modulation using digital micromirror device requires optimization of experimental parameters according to the target complex wave and its properties related with spatial resolution of quantization levels. The mathematical and physical principles that enable independent manipulation of phase and amplitude were described and discussed in detail. Moreover, we have discussed restrictions related to the distribution of the original information capacity of the encoding binary pattern and demonstrated that trade-off between the spatial resolution of the encoded complex wave and its quantization should be achieved in dependence on the target complex wave. It is shown that increasing one of these parameters results in an inevitable decrease in the other. In addition, we have proposed an approach for the optimization of the generated DMD binary patterns for each amplitude and phase distribution. The approach is based on a simulation of the modulated complex wave generation and takes into account the requirements of the target distribution to the quantization and spatial resolution.

The main applications of DMD for wavefront modulation, in which it is likely to be beneficial to maximize quantization or spatial resolution, are highlighted. Being optimized with the best experimental parameters, wavefront manipulation using DMD enables the accurate independent modulation of amplitude and phase distributions, and provides outstanding opportunities in various biomedical and technological applications. This technique is promising for implementation in various fields and applications, for instance, in the fast generation of shaped beams ${ }^{55}$ or in studies of scattering media, in particular, for modulation of ultrashort laser irradiation ${ }^{56,57}$. However, the best results can be achieved only when the DMD pattern generation algorithm is optimized for certain types of amplitude and phase images, specific for a given application.

\section{Declaration of Competing Interest}

None of the authors have any conflicts to declare.

\section{Acknowledgements}

A.B. and N.P. thank Russian Foundation for Basic Research (18-32-20215). 


\section{References}

1. Booth, M., Andrade, D., Burke, D., Patton, B. \& Zurauskas, M. Aberrations and adaptive optics in super-resolution microscopy. Microscopy. 64, 251-261, DOI: 10.1093/jmicro/dfv033 (2015).

2. Dickey, F. M. Laser beam shaping: theory and techniques (CRC press, 2018).

3. Ouadghiri-Idrissi, I. et al. Arbitrary shaping of on-axis amplitude of femtosecond bessel beams with a single phase-only spatial light modulator. Opt. Express. 24, 11495-11504, DOI: 10.1364/OE.24.011495 (2016).

4. Yu, H., Lee, K. \& Park, Y. Ultrahigh enhancement of light focusing through disordered media controlled by mega-pixel modes. Opt. Express. 25, 8036-8047, DOI: 10.1364/OE.25.008036 (2017).

5. Horstmeyer, R., Ruan, H. \& Yang, C. Guidestar-assisted wavefront-shaping methods for focusing light into biological tissue. Nat. Photonics. 9, 563, DOI: 10.1038/nphoton.2015.140 (2015).

6. Coyotl-Ocelotl, B., Porras-Aguilar, R., Ramos-Garcia, R. \& Ramirez-San-Juan, J. C. Implementation of focusing and redirecting light through highly scattering media. In SPECKLE 2015: VI International Conference on Speckle Metrology, vol. 9660, 355 - 359, DOI: 10.1117/12.2202820. International Society for Optics and Photonics (SPIE, 2015).

7. Chlipala, M. \& Kozacki, T. Color led dmd holographic display with high resolution across large depth. Opt. Lett. 44, 4255-4258, DOI: 10.1364/ol.44.004255 (2019).

8. Mirhosseini, M. et al. High-dimensional quantum cryptography with twisted light. New J. Phys. 17, 033033, DOI: 10.1088/1367-2630/17/3/033033 (2015).

9. Aulbach, L., Bloise, F. S., Lu, M. \& Koch, A. W. Non-contact surface roughness measurement by implementation of a spatial light modulator. Sensors. 17, DOI: 10.3390/s17030596 (2017).

10. Gao, L., Liang, J., Li, C. \& Wang, L. V. Single-shot compressed ultrafast photography at one hundred billion frames per second. Nature 516, 74, DOI: 10.1038/nature14005 (2014).

11. Yoon, T., Kim, C.-S., Kim, K. \& Choi, J.-r. Emerging applications of digital micromirror devices in biophotonic fields. Opt. Laser. Technol. 104, 17-25, DOI: 10.1016/j.optlastec.2018.02.005 (2018).

12. Rohwetter, P., Queißer, M., Stelmaszczyk, K., Fechner, M. \& Wöste, L. Laser multiple filamentation control in air using a smooth phase mask. Phys. Rev. A. 77, 013812, DOI: 10.1103/PhysRevA.77.013812 (2008).

13. Wang, W., Xie, Y., Popa, B.-I. \& Cummer, S. A. Subwavelength diffractive acoustics and wavefront manipulation with a reflective acoustic metasurface. J Appl Phys 120, 195103, DOI: 10.1063/1.4967738 (2016).

14. Salter, P. S. \& Booth, M. J. Adaptive optics in laser processing. Light. Sci. Appl. 8, 110, DOI: 10.1038/s41377-019-0215-1 (2019).

15. Wu, Z. \& Wang, X. DMD Mask Construction to Suppress Blocky Structural Artifacts for Medium Wave Infrared Focal Plane Array-Based Compressive Imaging. Sensors (Basel, Switzerland). 20, DOI: 10.3390/s20030900 (2020).

16. He, J., Dong, T., Chi, B. \& Zhang, Y. Metasurfaces for Terahertz Wavefront Modulation: a Review. J. Infrared Millim. TE. DOI: 10.1007/s10762-020-00677-3 (2020).

17. Zhou, G., Lim, Z. H., Qi, Y. \& Zhou, G. Single-Pixel MEMS Imaging Systems. Micromachines. 11, 219, DOI: 10.3390/mi11020219 (2020).

18. Zlokazov, E. Methods and algorithms for computer synthesis of holographic elements to obtain a complex impulse response of optical information processing systems based on modern spatial light modulators. Quantum Elec. 50, 643-652, DOI: 10.1070/QEL17291 (2020).

19. Yu, Z., Li, H. \& Lai, P. Wavefront shaping and its application to enhance photoacoustic imaging. Appl. Sci. 7, 1320, DOI: 10.3390/app7121320 (2017).

20. Turtaev, S. et al. Comparison of nematic liquid-crystal and dmd based spatial light modulation in complex photonics. Opt. Express. 25, 29874-29884, DOI: 10.1364/OE.25.029874 (2017).

21. Ren, Y., Rong-de, L. \& Gong, L. Tailoring light with a digital micromirror device. Ann. Phys. 527, 447-470, DOI: 10.1002/andp.201500111 (2015).

22. Park, M.-c. et al. Properties of DMDs for holographic displays. J. Mod. Opt. 62, 1600-1607, DOI: 10.1080/09500340. 2015.1054445 (2015).

23. Kalyoncu, S. K., Huang, Y., Song, Q. \& Boyraz, O. Fast Arbitrary Waveform Generation by Using Digital Micromirror Arrays. IEEE Phot. J. 5, 5500207, DOI: 10.1109/JPHOT.2013.2238664 (2013). 
24. Feng, W., Zhang, F., Wang, W., Xing, W. \& Qu, X. Digital micromirror device camera with per-pixel coded exposure for high dynamic range imaging. Appl. Opt. 56, 3831-3840, DOI: 10.1364/AO.56.003831 (2017).

25. Wen, C. et al. Spatially resolved random-access pump-probe microscopy based on binary holography. Opt. Lett. 44, 4083-4086, DOI: 10.1364/OL.44.004083 (2019).

26. Pan, Y., Liu, J., Li, X. \& Wang, Y. A Review of Dynamic Holographic Three-Dimensional Display: Algorithms, Devices, and Systems. IEEE T. Ind. Inform. 12, 1599-1610, DOI: 10.1109/TII.2015.2496304 (2016).

27. Tsang, P., Poon, T.-C., Cheung, W.-K. \& Liu, J.-P. Computer generation of binary fresnel holography. Appl. Opt. 50, B88-B95, DOI: 10.1364/AO.50.000B88 (2011).

28. Cheremkhin, P. A. \& Kurbatova, E. A. Comparative appraisal of global and local thresholding methods for binarisation of off-axis digital holograms. Opt. Laser. Eng. 115, 119-130, DOI: 10.1016/j.optlaseng.2018.11.019 (2019).

29. Chhetri, B. B., Yang, S. \& Shimomura, T. Iterative stepwise binarization of digital amplitude holograms with added energy to the signal window. Opt. Eng. 40, 2718-2725, DOI: 10.1117/1.1419019 (2001).

30. Holland, N. C., Stuart, D., Barter, O. \& Kuhn, A. G. Benchmarking modern algorithms to holographically create optical tweezers for laser-cooled atoms. J. Mod. Opt. 65, 2133 - 2141 (2018).

31. Yang, G., Jiao, S., Liu, J.-P., Lei, T. \& Yuan, X. Error diffusion method with optimized weighting coefficients for binary hologram generation. Appl. Opt. 58, 5547-5555, DOI: 10.1364/AO.58.005547 (2019).

32. Goorden, S. A., Bertolotti, J. \& Mosk, A. P. Superpixel-based spatial amplitude and phase modulation using a digital micromirror device. Opt. Express. 22, 17999-18009, DOI: 10.1364/OE.22.017999 (2014).

33. Lee, W. H. Binary synthetic holograms. Appl. Opt. 13, 1677-1682, DOI: 10.1364/AO.13.001677 (1974).

34. Gu, C., Zhang, D., Chang, Y. \& Chen, S.-C. Digital micromirror device-based ultrafast pulse shaping for femtosecond laser. Opt. Lett. 40, 2870-2873, DOI: 10.1364/OL.40.002870 (2015).

35. Conkey, D. B., Caravaca-Aguirre, A. M. \& Piestun, R. High-speed scattering medium characterization with application to focusing light through turbid media. Opt. Express. 20, 1733, DOI: 10.1364/oe.20.001733 (2012).

36. Reimers, J., Bauer, A., Thompson, K. P. \& Rolland, J. P. Freeform spectrometer enabling increased compactness. Light. Sci. Appl. 6, e17026-e17026, DOI: 10.1038/lsa.2017.26 (2017).

37. Zhang, Z., Wang, X., Zheng, G. \& Zhong, J. Fast Fourier single-pixel imaging via binary illumination. Sci. Rep. 7, 12029, DOI: $10.1038 / \mathrm{s} 41598-017-12228-3$ (2017).

38. Hirvonen, L. M., Wicker, K., Mandula, O. \& Heintzmann, R. Structured illumination microscopy of a living cell. Eur. Biophys. J. 38, 807-812 (2009).

39. Sandmeyer, A. et al. Dmd-based super-resolution structured illumination microscopy visualizes live cell dynamics at high speed and low cost. bioRxiv 797670 (2019).

40. Zhu, P., Fajardo, O., Shum, J., Schärer, Y.-P. Z. \& Friedrich, R. W. High-resolution optical control of spatiotemporal neuronal activity patterns in zebrafish using a digital micromirror device. Nat. Protoc. 7, 1410-1425 (2012).

41. Patterson, G. H. \& Lippincott-Schwartz, J. Selective photolabeling of proteins using photoactivatable gfp. Methods. 32, 445-450 (2004).

42. Degtyarev, S. A., Porfirev, A. P. \& Khonina, S. N. Zernike basis-matched multi-order diffractive optical elements for wavefront weak aberrations analysis. In Saratov Fall Meeting 2016: Laser Physics and Photonics XVII; and Computational Biophysics and Analysis of Biomedical Data III, vol. 10337, 103370Q (International Society for Optics and Photonics, 2017).

43. Pozzi, P. et al. High speed wavefront sensorless aberration correction in digital micromirror based confocal microscopy. Opt. Express. 25, 949-959 (2017).

44. Yoon, J., Kim, K. \& Park, W. Modulated grayscale uv pattern for uniform photopolymerization based on a digital micromirror device system. Appl. Phys. Lett. 111, 033505 (2017).

45. Shevkunov, I., Georgieva, A., Belashov, A. \& Petrov, N. V. Ptychography with DMD-based complex-valued probe. In Imaging and Applied Optics 2021, DM6C.6 (2021).

46. Liebling, M., Blu, T. \& Unser, M. Complex-wave retrieval from a single off-axis hologram. J. Opt. Soc. Am. A. 21, 367-377, DOI: 10.1364/JOSAA.21.000367 (2004). 
47. Katkovnik, V., Shevkunov, I. A., Petrov, N. V. \& Egiazarian, K. Wavefront reconstruction in digital off-axis holography via sparse coding of amplitude and absolute phase. Opt. Lett. 40, 2417-2420, DOI: 10.1364/OL.40.002417 (2015).

48. Belashov, A., Petrov, N. \& Semenova, I. Digital off-axis holographic interferometry with simulated wavefront. Opt. Express. 22, 28363-28376 (2014).

49. Dudley, A., Milione, G., Alfano, R. R. \& Forbes, A. All-digital wavefront sensing for structured light beams. Opt. Express. 22, 14031, DOI: 10.1364/OE.22.014031 (2014).

50. Ding, Y. et al. Wavefront shaping for fast focusing light through scattering media based on parallel wavefront optimization and superpixel method. Chin. Phys. Lett. 37, 024202, DOI: 10.1088/0256-307x/37/2/024202 (2020).

51. Falaggis, K., Kozacki, T. \& Kujawinska, M. Hybrid single-beam reconstruction technique for slow and fast varying wave fields. Opt. Lett. 40, 2509-2512, DOI: 10.1364/OL.40.002509 (2015).

52. Anh, N. T. L., Nhat, V. Q. \& Lee, G.-S. Object segmentation based on location information for level set method. In Proceedings of the 7th International Conference on Ubiquitous Information Management and Communication, ICUIMC '13, DOI: 10.1145/2448556.2448643 (Association for Computing Machinery, New York, NY, USA, 2013).

53. Hornbeck, L. J. Digital light processing and mems: reflecting the digital display needs of the networked society. In Micro-optical Technologies for Measurement, Sensors, and Microsystems, vol. 2783, 2-13 (International Society for Optics and Photonics, 1996).

54. Kim, J. U., Choi, H., Park, Y. \& Shin, J. Finite-difference time-domain analysis of increased penetration depth in optical coherence tomography by wavefront shaping. Biomed. Opt. Express. 9, 3883-3897, DOI: 10.1364/BOE.9.003883 (2018).

55. Mirhosseini, M. et al. Rapid generation of light beams carrying orbital angular momentum. Opt. Express. 21, 30196-30203, DOI: 10.1364/OE.21.030196 (2013).

56. Petrov, N. V., Goryunov, A. E. \& Pavlov, P. V. Investigation of interaction of structured illumination with random scattering media. Proc. SPIE. 9205, 92050T, DOI: 10.1117/12.2061328 (2014).

57. Belashov, A. V., Kulya, M. S., Balbekin, N. S., Gorodetsky, A. \& Petrov, N. V. Effect of object thickness on ultrashort pulse diffraction. Appl. Opt. 58, 9434, DOI: 10.1364/AO.58.009434 (2019). 


\section{Supplementary Files}

This is a list of supplementary files associated with this preprint. Click to download.

- Supplementary1.pdf 